\title{
Detection of torque teno sus virus infection in Indian pigs
}

Vinutha Subramanyam ${ }^{1,2}$, Divakar Hemadri ${ }^{1}$, Shashidhara Phani Kashyap ${ }^{1,2}$, Jagadish Hiremath ${ }^{1}$, Nagendra Nath Barman ${ }^{3}$, Esther Lalzoliani Ralte ${ }^{4}$, Sharanagouda S. Patil ${ }^{1}$, Kuralayanapalya P. Suresh ${ }^{1}$ and Habibur Rahaman ${ }^{1,5}$

1. ICAR-National Institute of Veterinary Epidemiology and Disease Informatics, Yelahanka, Bengaluru, Karnataka, India; 2. Department of Microbiology and Biotechnology, Jain University, Bengaluru, Karnataka, India; 3. Department of Veterinary Microbiology, College of Veterinary Science, Assam Agriculture University, Guwahati, Assam, India; 4. State Disease Investigation Laboratory, Directorate of Animal Husbandry and Veterinary, Aizawl, Mizoram, India; 5. Regional Representative for South Asia, International Livestock Research Institute, New Delhi, India.

Corresponding author: Divakar Hemadri, e-mail: divakar.hemadri@icar.gov.in

Co-authors: VS: viny.naidu89@gmail.com, SPK: phanikashyaps@gmail.com, JH: jagsvet@gmail.com,

NNB: nnbarman@gmail.com, ELR: esralte185@yahoo.com, SSP: ss.patil@icar.gov.in, KPS: sureshkp97@gmail.com, HR: hricar@gmail.com

Received: 31-05-2019, Accepted: 16-08-2019, Published online: 25-09-2019

doi: 10.14202/vetworld.2019.1467-1471 How to cite this article: Subramanyam V, Hemadri D, Kashyap SP, Hiremath J, Barman NN, Ralte EL, Patil SS, Suresh KP, Rahaman H. (2019) Detection of torque teno sus virus infection in Indian pigs, Veterinary World, 12(9): 1467-1471.

\begin{abstract}
Background and Aim: Torque teno viruses (TTVs) are circular, single-stranded DNA viruses, which infect a wide range of animals including livestock and companion animals. Swine TTVs (torque teno sus viruses [TTSuVs]) are thought to act as a primary or coinfecting pathogen in pathological conditions such as porcine dermatitis and nephropathy syndrome and postweaning multisystemic wasting syndrome. So far, the presence of the virus has not been reported in India. Considering that TTSuVs have the potential to cross the species barrier into humans and that pork consumption is common in North-Eastern states of India, the current study aims to investigate the presence of TTSuV in the Indian pig population.
\end{abstract}

Materials and Methods: A total of 416 samples were collected during 2014-2018, from both apparently healthy pigs and also from pigs suspected of having died from classical swine fever and/or porcine reproductive and respiratory syndrome. These samples were screened for TTSuV infection by polymerase chain reaction (PCR) and DNA sequencing techniques.

Results: The presence of the virus was confirmed in 110 samples from 12 different states of India. Phylogenetic analysis of the nucleotide sequences obtained from the PCR products indicated the presence of viruses of both Iotatorquevirus and Kappatorquevirus genera in India.

Conclusion: The study is the first report on the presence of TTSuVs in India and highlights the circulation of both genera of the virus in the country.

Keywords: detection, India, phylogeny, polymerase chain reaction, torque teno virus.

\section{Introduction}

Torque teno viruses (TTVs) are circular, single-stranded DNA viruses, which infect a wide range of animals including livestock and companion animals [1-3]. Natural infection of pigs with TTV was first described in 1999 [1]. However, a retrospective study from Spain [4] revealed that the virus had been present since at least 1985. Based on nucleotide (NT) sequence homology, TTVs of swine, known as torque teno sus viruses (TTSuVs), are divided into two genera; Iotatorquevirus (TTSuV1) and Kappatorquevirus (TTSuVk2), with two species in each genus [5].

At present, no clinical signs are specifically associated with TTSuV infection, and no clinicopathological experimental studies involving

Copyright: Subramanyam, et al. Open Access. This article is distributed under the terms of the Creative Commons Attribution 4.0 International License (http://creativecommons.org/licenses/ by/4.0/), which permits unrestricted use, distribution, and reproduction in any medium, provided you give appropriate credit to the original author(s) and the source, provide a link to the Creative Commons license, and indicate if changes were made. The Creative Commons Public Domain Dedication waiver (http:// creativecommons.org/publicdomain/zero/1.0/) applies to the data made available in this article, unless otherwise stated. conventional pigs have been done due to the lack of readily available isolates [6]. However, there is a report that inoculation of gnotobiotic pigs with TTSuV1-containing tissue homogenate caused mild interstitial pneumonia, transient thymic atrophy, membranous glomerulonephropathy, and modest lymphocytic-to-histiocytic liver infiltrates [7]. In addition, there are studies in gnotobiotic pigs, which indicated that swine TTVs, TTSuV1 in particular, can act as a primary or coinfecting pathogen in pathological conditions such as porcine dermatitis and nephropathy syndrome $[7,8]$ and post-weaning multisystemic wasting syndrome $[8,9]$. Further, there is a report that the rate of detection of TTSuVs in pigs with clinical signs of the coinfecting pathogens is roughly double than that of clinically healthy pigs [10]. In addition, recent studies have reported a high rate of coinfection between TTSuVs and porcine circovirus (PCV)-associated disease including in PCV3 infection $[9,11,12]$. In India, there are few studies on the presence and distribution of TTVs in the human population [13-15]; nevertheless, the rate of TTV detection has been found to be significantly higher $(92.8 \%)$ for 
non-A-E hepatitis group [16]. Until now, there has not been a report on the presence of TTSuVs in animals including pigs in India.

Considering that TTSuVs have the potential to cross the species barrier and infect humans [17] and that pork consumption is common in North-Eastern states of India, this study aims to report the presence of TTSuV in India, based on the screening of tissue specimens/blood/serum/swab samples collected from different parts of the country.

\section{Materials and Methods}

\section{Ethical approval}

This study did not involve the use of live animals, and hence, ethical approval was not required.

\section{Samples}

A total of 416 samples were collected during 2014-2018, from both apparently healthy pigs and also from pigs suspected of having died of classical swine fever and/or porcine reproductive and respiratory syndrome.

\section{DNA extraction}

DNA extraction was performed on individual samples using a commercial DNA extraction kit (DNeasy Blood and Tissue Kit, QIAGEN, Germany) according to the manufacturer's instructions.

\section{Polymerase chain reaction (PCR) and DNA sequencing}

The PCR was performed using the primers specific for TTSuV1 [18] and TTSuVk2 [19], which targeted a portion of the non-coding region of each viral genome and was expected to produce amplicons of approximately 290 and 230 bases, respectively. Each reaction consisted of $3 \mu \mathrm{LNA}$ as template, $1 \times \mathrm{PCR}$ buffer, $2.5 \mathrm{mM} \mathrm{MgCl}_{2}, 1.0 \mathrm{mM}$ dNTP, 10 pmol forward and reverse primer, $0.25 \mathrm{U}$ Taq DNA polymerase (New England BioLabs, USA), and nuclease-free water. The amplification was performed with the following reaction conditions: A $10 \mathrm{~min}$ initial denaturation step at $95^{\circ} \mathrm{C}$, followed by 35 cycles at $95^{\circ} \mathrm{C}$ for $30 \mathrm{~s}, 55^{\circ} \mathrm{C}$ for $30 \mathrm{~s}$, and $72^{\circ} \mathrm{C}$ for $30 \mathrm{~s}$, finishing with 1 cycle for $10 \mathrm{~min}$ at $72^{\circ} \mathrm{C}$. The PCR products were visualized on a $1.5 \%$ agarose gel. The PCR-positive products were purified using the QIAquick PCR Purification Kit (Qiagen, Hilden, Germany) according to the manufacturer's instructions and eluted in $30 \mu \mathrm{l}$ of elution buffer. The representative amplified products were cloned into the pGEM-T vector (Promega, USA) and subjected to NT sequencing using M13 primers (Eurofins Scientific, India).

\section{Phylogenetic analysis}

Phylogenetic analysis was carried out using MEGA X software [20]. Briefly, the NT sequences of TTSuVs generated in the present study were aligned and analyzed with the prototype sequences of all of the four presently defined species of the virus and several additional sequences available in the NCBI GenBank database.

\section{Results}

PCR

TTSuV was detected in pigs from 12 of the 16 different states investigated and a total of 110 samples were positive of the 416 samples screened. Of those samples which were positive, 40 were positive for TTSuV1, while 70 were positive for TTSuVk2. The details of the samples are given in Table-1.

\section{Phylogenetic analysis}

Phylogenetic analysis of the NT sequences obtained from the above PCR products of TTSuV is shown in Figure-1a and b. From Figure-1b, it can be seen that all the NT sequenced TTSuVs of the Kappatorquevirus genus in our study belong to the species, TTSuVk2a; on the contrary, the species identity of the TTSuVs of the Iotatorquevirus genus circulating in India could not be determined as their

Table-1: Details of samples screened for TTSuV infection in domestic pigs.

\begin{tabular}{|c|c|c|c|c|c|c|c|c|}
\hline \multirow[t]{2}{*}{ S. No. } & \multirow[t]{2}{*}{ State } & \multirow{2}{*}{$\begin{array}{l}\text { Number of } \\
\text { screened }\end{array}$} & \multicolumn{4}{|c|}{ Type of material } & \multirow{2}{*}{$\begin{array}{l}\text { Number of } \\
\text { TTSuV1 positive }\end{array}$} & \multirow{2}{*}{$\begin{array}{l}\text { Number of } \\
\text { TTSuV2 positive }\end{array}$} \\
\hline & & & Tissue & Blood & Nasal swab & Serum & & \\
\hline 1 & Andhra Pradesh & 6 & 0 & 6 & 0 & 0 & 0 & 0 \\
\hline 2 & Arunachal Pradesh & 21 & 0 & 0 & 0 & 21 & 0 & 0 \\
\hline 3 & Assam & 20 & 14 & 6 & 0 & 0 & 1 & 1 \\
\hline 4 & Chhattisgarh & 10 & 0 & 0 & 0 & 10 & 2 & 2 \\
\hline 5 & Goa & 38 & 13 & 4 & 13 & 8 & 4 & 6 \\
\hline 6 & Karnataka & 105 & 28 & 66 & 0 & 11 & 14 & 23 \\
\hline 7 & Kerala & 26 & 2 & 0 & 0 & 24 & 1 & 1 \\
\hline 8 & Madhya Pradesh & 27 & 6 & 2 & 0 & 19 & 1 & 2 \\
\hline 9 & Maharashtra & 37 & 11 & 2 & 3 & 21 & 4 & 14 \\
\hline 10 & Manipur & 14 & 0 & 0 & 0 & 14 & 0 & 0 \\
\hline 11 & Meghalaya & 4 & 1 & 0 & 0 & 3 & 1 & 1 \\
\hline 12 & Mizoram & 33 & 13 & 13 & 0 & 7 & 3 & 3 \\
\hline 13 & Odisha & 7 & 7 & 0 & 0 & 0 & 0 & 2 \\
\hline 14 & Punjab & 13 & 0 & 3 & 7 & 3 & 0 & 0 \\
\hline 15 & Sikkim & 42 & 0 & 42 & 0 & 0 & 6 & 7 \\
\hline 16 & Telangana & 13 & 7 & 6 & 0 & 0 & 3 & 8 \\
\hline Total & & 416 & 102 & 150 & 23 & 141 & 40 & 70 \\
\hline
\end{tabular}

TTSuV=Torque Teno Sus Virus 


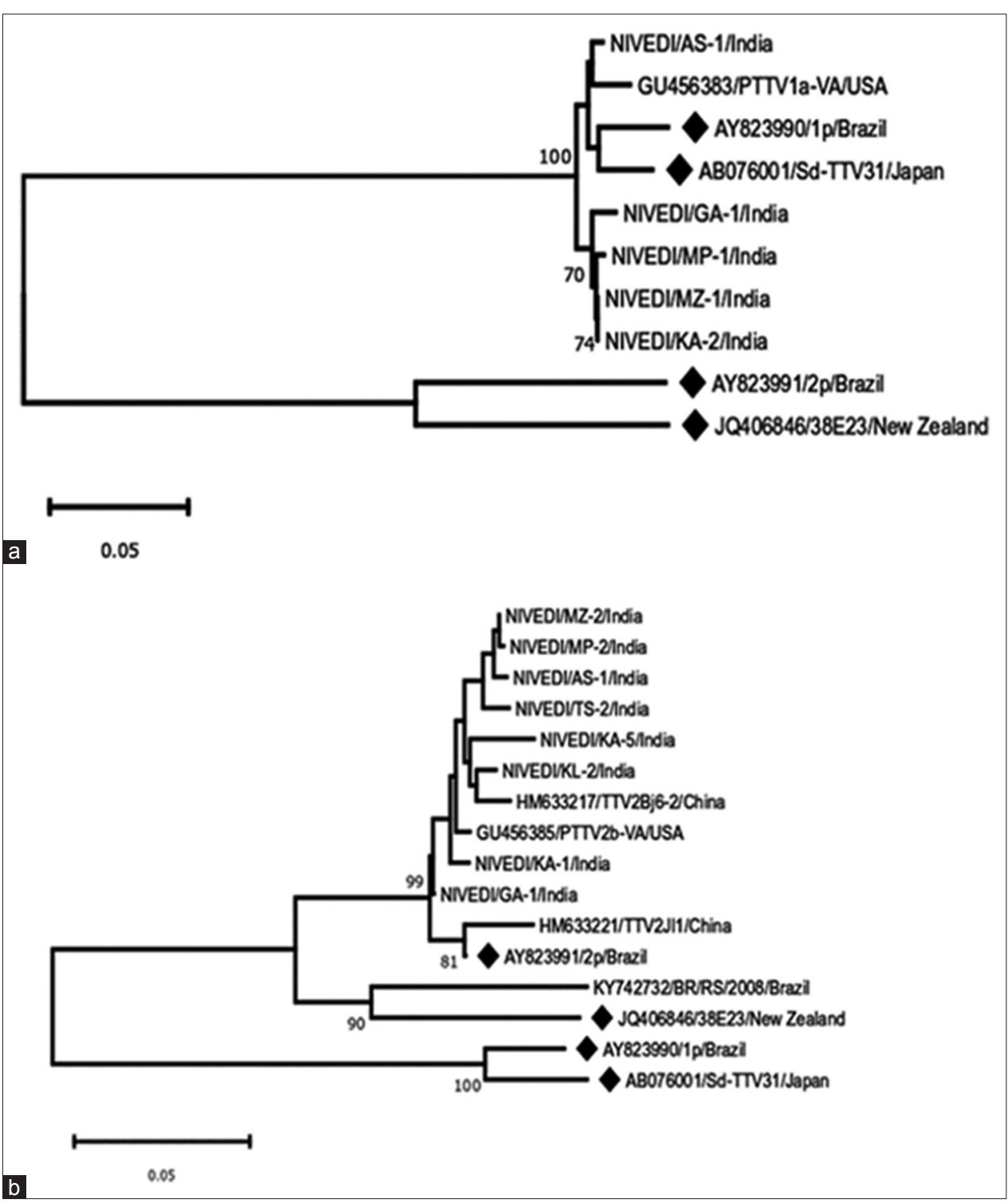

Figure-1: Neighbor-joining tree showing the grouping of Indian torque teno sus viruses (TTSuVs) with the prototype species ( $\bullet$ ) of the genus, Iotatorquevirus (a): TTSuV1a (AB076001) and TTSuV1b (AY823990) and Kappatorquevirus (b): TTSuV K2a (AY823991) and TTSuV K2b (JQ406846). The percentage of replicate trees in which the associated taxa clustered together in the bootstrap test (1000 replicates) is shown next to the branches. The tree was drawn to scale, with branch lengths in the same units as those of the evolutionary distances used to infer the phylogenetic tree. The evolutionary distances were computed using the p-distance method and are in the units of the number of base differences per site.

grouping on the phylogenetic tree (Figure-1a) was not sufficiently resolved.

\section{Discussion}

In all, of the 416 samples screened for TTSuV, 110 samples were positive, of which 40 were positive for TTSuV1, while 70 were positive for TTSuVk2. Earlier, we made attempts to amplify TTSuV1 using the primer pair reported by Kekarainen [19]. However, these primers failed to detect TTSuV1 in our samples. In contrast, TTSuV1 was successfully detected in our samples using the primer pair reported by Ozawa et al. [18]. A comparison of the primer sequences revealed that the reverse primer used by Ozawa et al. [18] though almost identical to that of Kekarainen [19] differs by three NT bases at the 3' end. The difference in the NT composition of Indian TTSuV1 in these positions could be the reason for the failure in our earlier attempts. NT sequencing of the representative PCR-amplified products further confirmed that these were, in fact, specific for TTSuVs. Among the samples screened from 16 Indian states (Table-1), it could be seen that samples from 10 states were positive for both the TTSuV genera. Samples from the states of Odisha and Kerala were negative for TTSuV1, while those from Andhra Pradesh, Arunachal Pradesh, Sikkim, and Punjab were negative in both the genus-specific PCRs. Considering the variable prevalence (17-100\%) of TTSuVs globally [6], screening of a larger number of samples from the above states would be required to accurately assess their prevalence in these regions. 
It is worth noting that although TTSuVs have been detected in different organs, relatively high detection rates have been found in the kidneys of apparently healthy slaughterhouse aged pigs [21]. Among the various types of samples screened, 35.3, 28.7, 16.3, and $34.8 \%$ of tissue specimens, blood, serum, and nasal swabs, respectively, were positive for the presence of TTSuVs. Phylogenetic analysis of the NT sequences obtained from the above PCR products of TTSuV is shown in Figure-1a and b. From Figure-1b, it can be seen that all of the NT sequenced TTSuVs of the Kappatorquevirus genus in our study belong to the species, TTSuVk2a; on the contrary, the species identity of TTSuVs of the Iotatorquevirus genus circulating in India could not be determined as their grouping on the phylogenetic tree (Figure-1a) was not resolved sufficiently. It has been reported that non-coding regions contain NT sequences which are highly conserved among TTVs infecting humans, non-human primates, and other animals [2], as this region harbors regulatory sequences important in replication and transcription [22], and promoter and enhancer elements that exhibit different transcriptional activities in different cell lines $[23,24]$. Here, we used primers located in non-coding regions, wherein sequence homologies of $91-97 \%$ and $93-99 \%$ were noted for the TTSuV1 and TTSuVk2 genogroups, respectively [18]. Therefore, it is quite plausible that the NT divergence observed in the PCR-amplified region (260-290 bases) was not sufficient for species classification of Iotatorquevirus by phylogenic techniques.

\section{Conclusion}

In the present study, we have screened specimens from diverse and distant geographical regions of India and the detection of TTSuVs in these specimens indicated the widespread presence of these viruses across the country. Further studies involving more genomic sequences of the coding region are required to provide a more accurate assessment of the prevalence of TTSuVs circulating in India. Nevertheless, the present study has provided us with first-hand knowledge of the circulation of TTSuVs in India and also that the viruses of both genera are prevalent.

\section{Authors' Contributions}

VS, DH, KPS, and HR conceived and designed the experiments. DH wrote the manuscript, while $\mathrm{JH}$ and SSP assisted in writing the manuscript. VS, SPK, NNB, and ELR performed the experiments. All authors read and approved the final manuscript.

\section{Acknowledgments}

The authors are thankful to the Department of Biotechnology, Ministry of Science and Technology, Government of India, for funding (DBT-NER/ LIVS/11/2012). Indian Council of Agricultural Research (ICAR), New Delhi, and ICAR-National Institute of Veterinary Epidemiology and Disease
Informatics (ICAR-NIVEDI), Bengaluru, Karnataka, India, are acknowledged for providing the necessary facilities and administrative support. We profusely thank Dr. Jeff Buttler, AAHL, CSIRO, Australia, and Dr. Yashawant S. L., USA for critical reading of the manuscript and linguistic corrections.

\section{Competing Interests}

The authors declare that they have no competing interests.

\section{Publisher's Note}

Veterinary World remains neutral with regard to jurisdictional claims in published institutional affiliation.

\section{References}

1. Leary, T., Desai, S., Chalmers, M., Erker, J. and Mushahwar, I. (1999). Improved detection systems for TT virus reveal high prevalence in humans, non-human primates and farm animals. J. Gen. Virol., 80(8): 2115-2120.

2. Okamoto, H., Takahashi, M., Nishizawa, T., Tawara, A., Fukai, K., Muramatsu, U., Naito, Y. and Yoshikawa, A. (2002) Genomic characterization of TT viruses (TTVs) in pigs, cats and dogs and their relatedness with species-specific TTVs in primates and tupaiahs. J. Gen. Virol., 83(6):1291-1297.

3. Fahsbender, E., Burns, J.M., Kim, S., Kraberger, S., Frankfurter, G., Eilers, A.A., Shero, M.R., Beltran, R., Kirkham, A., McCorkell, R., Berngartt, R.K., Male, M.F., Ballard, G., Ainley, D.G., Breitbart, M. and Varsani, A. (2017) Diverse and highly recombinant Anelloviruses associated with Weddell seals in Antarctica. Virus Evol., 3(1): vex017.

4. Segalés, J., Martinéz-Guinó, L., Cortey, M., Navarro, N., Huerta, E., Sibila, M., Pijols, J. and Kekarainen, T. (2009) Retrospective study on swine torque teno virus genogroups 1 and 2 infection from 1985 to 2005 in Spain. Vet. Microbiol., 134(3-4): 199-207.

5. Lefkowitz, E.J., Dempsey, D.M., Hendrickson, R.C., Orton, R.J., Siddell, S.G. and Smith, D.B. (2018) Virus taxonomy: The database of the international committee on taxonomy of viruses (ICTV). Nucleic Acids Res., 46(D1): D708-D717.

6. Segalés, J. and Kekarainen, T. (2019) Anelloviruses. In: Zimmerman, J.J., Karriker, L.A., Ramirez, A., Schwartz, K.J., Stevenson, G.W. and Zhang, J., editors. $11^{\text {th }}$ ed. John Wiley and Sons, Inc. USA. p453-456.

7. Krakowka, S. and Ellis, J. (2008) Evaluation of the effects of porcine genogroup 1 torque teno virus in gnotobiotic swine. Am. J. Vet. Res., 69(12): 1623-1629.

8. Ellis, J., Allan, G. and Krakowka, S. (2008) Effect of coinfection with genogroup 1 porcine torque teno virus on porcine circovirus Type 2 associated postweaning multisystemic wasting syndrome in gnotobiotic pigs. Am. J. Vet. Res., 69(12): 1608-1614.

9. Vargas-Ruiz, A., García-Camacho, L.A., Ramírez-Alvarez, H., Rangel-Rodriguez, I.C., AlonsoMorales, R.A. and Sánchez-Betancourt, J.I. (2018) Molecular characterization of the ORF2 of Torque teno sus virus $1 \mathrm{a}$ and torque teno sus virus $1 \mathrm{~b}$ detected in cases of postweaning multisystemic wasting syndrome in Mexico. Transbound. Emerg. Dis., 65(6): 1-10.

10. Rammohan, L., Xue, L., Wang, C., Chittick, W., Ganesan, S. and Ramamoorthy, S. (2012) Increased prevalence of torque teno viruses in porcine respiratory disease complex affected pigs. Vet. Microbiol., 157(1-2): 61-68.

11. de Castro, A.M.M., Baldin, C.M., Favero, C.M., Gerber, P.F., Cipullo, R.I. and Richtzenhain, L.J. (2015) Torque teno 
sus virus 1 and 2 viral loads in feces of porcine circovirus 2-positive pigs. Acta Vet. Brno., 84(2): 91-95.

12. Ramos, N., Mirazo, S., Botto, G., Teixeira, T.F., Cibulski, S.P., Castro, G., Cabrera, K., Roehe, P.M. and Arbiza, J. (2018) High frequency and extensive genetic heterogeneity of TTSuV1 and TTSuVk2a in PCV2 infected and non-infected domestic pigs and wild boars from Uruguay. Vet. Microbiol., 224: 78-87.

13. Abraham, P., John, G., Raghuraman, S., Radhakrishnan, S., Thomas, P., Jacob, C. and Sridharan, G. (2003) GB virus $\mathrm{C} /$ hepatitis $\mathrm{G}$ virus and TT virus infections among high-risk renal transplant recipients in India. J. Clin. Virol., 28(1): 59-69.

14. Desai, M., Pal, R. and Banker, D. (2005) Molecular epidemiology and clinical implications of TT virus (TTV) infection in Indian subjects. J. Clin. Gastroenterol., 39(5): 422-429.

15. Asim, M., Singla, R., Gupta, R.K. and Kar, P. (2010) Clinical and molecular characterization of human TT virus in different liver diseases. Indian J. Med. Res., 131(4): 545-554.

16. Magu, S.K., Kalghatgi, A.T. and Bhagat, M.R. (2015) Incidence and clinical implication of TT virus in patients with hepatitis and its frequency in blood donors in India. Med. J. Armed Forces India, 71(4): 340-344.

17. Singh, G. and Ramamoorthy, S. (2018) Potential for the cross-species transmission of swine torque teno viruses. Vet. Microbiol., 215: 66-70.

18. Ozawa, M., Kawabata, T., Okuya, K., Nagano, K., Kanda, T., Kanazawa, N., Tsukiyama-Kohara, K., Taneno, A. and Deguchi, E. (2015) Full genome sequences of torque teno sus virus strains that coinfected a pig with postweaning multisystemic wasting syndrome in Japan: Implications for genetic diversity. Arch. Virol., 160(12): 3067-3074.

19. Kekarainen, T. (2006) Prevalence of swine Torque teno virus in post-weaning multisystemic wasting syndrome (PMWS)-affected and non-PMWS-affected pigs in Spain. J. Gen. Virol., 87(4): 833-837.

20. Kumar, S., Stecher, G., Li, M., Knyaz, C. and Tamura, K. (2018) MEGA X: Molecular evolutionary genetics analysis across computing platforms. Mol. Biol. Evol., 35(6): 1547-1549.

21. Ghosh, S., Soto, E., Illanes, O., Aung, M.S., Malik, Y.S., Kobayashi, N. and Fuentealba, C. (2018) High detection rates of Torque teno sus virus in co-infection with important viral pathogens in porcine kidneys on St. Kitts Island, Lesser Antilles. Transbound. Emerg. Dis., 65(5): 1175-1181.

22. Mankertz, A., Caliskan, R., Hattermann, K., Hillenbrand, B., Kurzendoerfer, P., Mueller, B., Schmitt, C., Steinfeldt, T. and Finsterbusch, T. (2004) Molecular biology of Porcine circovirus: Analyses of gene expression and viral replication. Vet. Microbiol., 98(2): 81-88.

23. Kamada, K., Kamahora, T., Kabat, P. and Hino, S. (2004) Transcriptional regulation of TT virus: Promoter and enhancer regions in the 1.2-kb noncoding region. Virology, 321(2): 341-348.

24. Suzuki, T., Suzuki, R., Li, J., Hijikata, M., Matsuda, M., Li, T.C., Matsuura, Y., Mishiro, S. and Miyamura, T. (2004) Identification of basal promoter and enhancer elements in an untranslated region of the TT virus genome. J. Virol., 78(19): 10820-10824.

$* * * * * * * *$ 